\title{
Shape sensitivity and reliability analyses of linear-elastic cracked structures
}

\author{
GUOFENG CHEN $^{1}$, SHARIF RAHMAN ${ }^{1, *}$ and YOUNG HO PARK ${ }^{2}$ \\ ${ }^{1}$ Department of Mechanical Engineering, The University of Iowa, Iowa City, IA 52242, U.S.A. $\left({ }^{*}\right.$ Author for \\ correspondence: Fax: (319) 335-5669; E-mail: rahman@engineering.uiowa.edu) \\ ${ }^{2}$ Department of Mechanical Engineering, New Mexico State University, Las Cruces, NM 88003, U.S.A.
}

Received 20 June 2000; accepted in revised form 31 July 2001

\begin{abstract}
This paper presents a new method for continuum-based shape sensitivity and reliability analyses of a crack in a homogeneous, isotropic, and linear-elastic body subject to mode-I loading. The method involves the material derivative concept of continuum mechanics, domain integral representation of the $J$-integral, and direct differentiation. Unlike virtual crack extension techniques, no mesh perturbation is needed in the proposed sensitivity analysis method. Since the governing variational equation is differentiated prior to the process of discretization, the resulting sensitivity equations are independent of any approximate numerical techniques, such as the finite element method, boundary element method, or others. Numerical results show that the maximum error in calculating the sensitivity of $J$ using the proposed method is less than three percent. Based on continuum sensitivities, the first-order reliability method was formulated to conduct probabilistic fracture-mechanics analysis. A numerical example is presented to illustrate the usefulness of the proposed sensitivity equations for probabilistic analysis. Since all gradients are calculated analytically, the reliability analysis of cracks can be performed efficiently.
\end{abstract}

Key words: Crack, energy release rate, $J$-integral, linear-elastic fracture mechanics, probabilistic fracture mechanics, reliability, shape sensitivity analysis, stress-intensity factor.

\section{Introduction}

In probabilistic fracture mechanics (PFM), the derivatives of stress-intensity factor (SIF) or energy release rate (ERR) are often required to predict the probability of fracture initiation and/or instability in cracked structures. For example, the first- and second-order reliability methods (FORM/SORM) (Madsen et al., 1986), frequently used in PFM (Grigoriu et al., 1990; Provan, 1987; Besterfield et al., 1990, 1991; Rahman, 1995, 2001; Rahman and Kim, 2000), require the gradient and Hessian of the performance function with respect to the crack length. In linear-elastic fracture mechanics (LEFM), the performance function builds on SIF. Hence, both first- and second-order derivatives of SIF are needed for probabilistic analysis. The calculation of these derivatives with respect to load and material parameters, which constitutes size-sensitivity analysis, is not unduly difficult. However, the evaluation of response derivatives with respect to crack size is a challenging task, since shape sensitivity analysis is required. Using a brute-force type finite-difference method to calculate shape sensitivities is often computationally expensive, since many repetitions of deterministic finite element analysis may be required for a complete reliability analysis. Therefore, an important requirement of PFM is to evaluate the rates of SIF and ERR accurately and efficiently.

Some methods have already appeared for predicting sensitivities of SIF or ERR. In 1988, Lin and Abel (1988) introduced a direct-integration approach of the virtual crack extension 
technique (deLorenzi, 1982, 1985;Haber and Koh, 1985; Barbero and Reddy, 1990) that employs variational formulation and the finite element method (FEM) to calculate the first-order derivative of SIF for a structure containing a single crack. Subsequently, Hwang et al. (1998) generalized this method to calculate both first- and second-order derivatives for structures involving multiple crack systems, axisymmetric stress state, and crack-face and thermal loading. A salient feature of this method is that SIFs and their derivatives can be evaluated in a single analysis. However, this method requires mesh perturbation - a fundamental requirement of all virtual crack extension techniques. For second-order derivatives, the number of elements surrounding the crack tip that are affected by mesh perturbation has a significant effect on the solution accuracy (Hwang et al., 1998). In 1992, Keum et al. (1992a, b) developed a boundary integral formulation for sensitivity with changing boundary conditions, and applied this method to calculate the energy release rate and stress intensity factors of two-dimensional linear-elastic cracked bodies. Recently, Feijóo et al. (Feijóo et al., 2000) applied the concepts of shape sensitivity analysis (Choi and Haug, 1983; Haug et al., 1986) to calculate the firstorder derivative of the potential energy. Since ERR is the first derivative of potential energy, this approach can be used to calculate the ERR without any mesh perturbation. Taroco (2000) later extended this approach to formulate the second-order sensitivity of the potential energy in predicting the first-order derivative of the ERR; however, this is a difficult task, since the calculation of second-order sensitivities of stress and strain is involved. It is worth mentioning that no numerical results of the sensitivity of ERR were reported by Taroco (2000).

Although development is still ongoing, a number of methods are available to estimate statistics of various fracture response and reliability. Most of these methods are based on LEFM using SIF as the primary crack-driving force (Grigoriu et al., 1990; Provan, 1987; Besterfield et al., 1990, 1991; Rahman, 1995, 2001; Rahman and Kim, 2000). For example, using SIFs from a deterministic LEFM code, Grigoriu et al. (1990) applied FORM/SORM algorithms to predict the probability of fracture initiation and the confidence interval of the direction of crack extension. The method can account for random loads, material properties, and crack geometry. However, the randomness in crack geometry was modeled by response surface approximations of SIFs as explicit functions of crack geometry. Similar responsesurface-based methods involving elastic-plastic fracture mechanics and the $J$-integral-based ductile tearing theory have also appeared (Rahman, 1995, 2000; Rahman and Kim, 2000). For example, a stochastic model based on an engineering approximation of the $J$-integral and FORM/SORM has been developed by Rahman and co-workers for fracture analysis of cracked tubular structures (Rahman, 1995). Based on this model, the probability of fracture initiation and subsequent fracture instability can be predicted under elastic-plastic conditions. The response surface approximation used in these PFM analyses significantly reduces complexity in calculating the derivatives of the SIF or the $J$-integral. Actually, this reduced complexity is a major reason why FORM/SORM algorithms have been successfully utilized in these methods. However, the usefulness of response-surface based methods is limited, since they cannot be applied for general fracture-mechanics analysis. Due to the complexity in crack geometry, external loads, and material behavior, more advanced computational tools, such as FEM or other numerical methods, must be employed to provide the necessary computational framework for analysis of general cracked structures. Although calculation of SIF, $J$, and other relevant fracture parameters by FEM is not unduly difficult, the evaluation of response derivatives or sensitivities, required by FORM/SORM analysis, is a challenging task. It is worth noting that a general probabilistic model integrating elastic-plastic finite element analysis and FORM/SORM with finite-difference-based sensitivities has appeared recently 


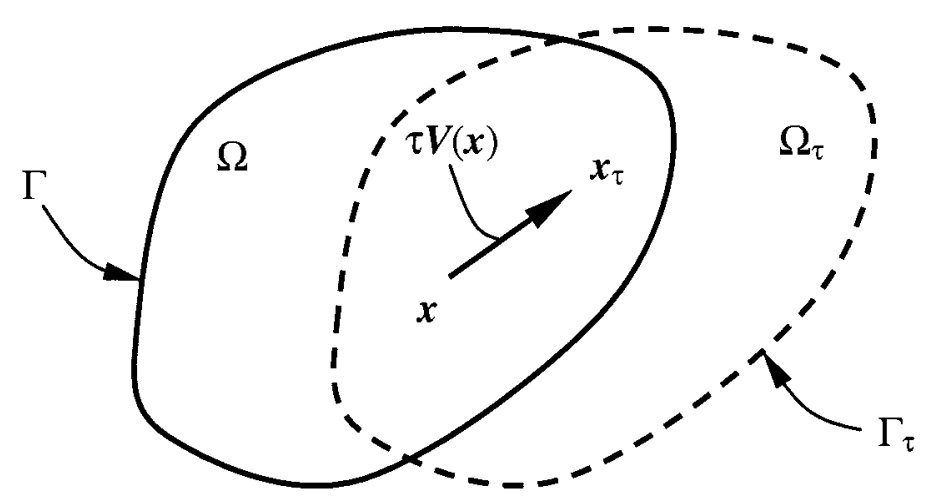

Figure 1. Variation of domain.

(Rahman and Kim, 2000), and has been used in validating failure probabilities predicted by response-surface-based PFM methods (Rahman, 2001).

This paper presents a new method for predicting the first-order sensitivity of the $J$-integral for a crack in a homogeneous, isotropic, and linear-elastic structure subject to mode-I loading conditions. The method involves the material derivative concept of continuum mechanics, domain integral representation of the $J$-integral, and direct differentiation. Several numerical examples are presented to calculate the first-order derivative of the $J$-integral, using the proposed method. The results from this method are compared with results from the analytical or the finite-difference method. Based on continuum sensitivities, the first-order reliability method is formulated to predict the stochastic response and reliability of cracked structures. A fracture reliability problem is also presented to illustrate the effectiveness of the proposed sensitivity equations.

\section{Shape sensitivity analysis}

\subsection{VELOCITY FIELD}

Consider a general three-dimensional body with a specific configuration, referred to as the reference configuration, with domain $\Omega$, boundary $\Gamma$, and a body material point identified by position vector $\mathbf{x} \in \Omega$. Consider the motion of the body from the configuration with domain $\Omega$ and boundary $\Gamma$ into another configuration with domain $\Omega_{\tau}$ and boundary $\Gamma_{\tau}$, as shown in Figure 1. This process can be expressed as

$$
\mathbf{T}: \mathbf{x} \rightarrow \mathbf{x}_{\tau}, \quad \mathbf{x} \in \Omega
$$

where $\boldsymbol{x}$ and $\boldsymbol{x}_{\tau}$ are the position vectors of a material point in the reference and perturbed configurations, respectively, $\boldsymbol{T}$ is a transformation mapping, and $\tau$ is a time-like parameter with

$$
\mathbf{x}_{\tau}=\boldsymbol{T}(\mathbf{x}, \tau), \Omega_{\tau}=\boldsymbol{T}(\Omega, \tau), \Gamma_{\tau}=\boldsymbol{T}(\Gamma, \tau) .
$$

A velocity field $\boldsymbol{V}$ can then be defined as 


$$
\boldsymbol{V}\left(\mathbf{x}_{\tau}, \tau\right)=\frac{\mathrm{d} \mathbf{x}_{\tau}}{\mathrm{d} \tau}=\frac{\mathrm{d} \mathbf{T}(\mathbf{x}, \tau)}{\mathrm{d} \tau}=\frac{\partial \boldsymbol{T}(\mathbf{x}, \tau)}{\partial \tau} .
$$

In the neighborhood of an initial time $\tau=0$, assuming a regularity hypothesis and ignoring high-order terms, $\boldsymbol{T}$ can be approximated by

$$
\boldsymbol{T}(\mathbf{x}, \tau)=\boldsymbol{T}(\mathbf{x}, 0)+\tau \frac{\partial \boldsymbol{T}(\mathbf{x}, 0)}{\partial \tau}+\mathrm{O}\left(\tau^{2}\right) \cong \mathbf{x}+\tau \boldsymbol{V}(\mathbf{x}, 0),
$$

where $\mathbf{x}=\boldsymbol{T}(\mathbf{x}, 0)$ and $\boldsymbol{V}(\mathbf{x})=\boldsymbol{V}(\mathbf{x}, 0)$.

\subsection{ShaPE SENSITIVITY ANALYSIS}

The variational governing equation for a structural component with domain $\Omega$ can be formulated as (Choi and Haug, 1983; Haug et al., 1986)

$$
a_{\Omega}(\mathbf{z}, \overline{\mathbf{z}})=\ell_{\Omega}(\overline{\mathbf{z}}), \text { for all } \overline{\mathbf{z}} \in \mathbf{Z}
$$

where $\mathbf{z}$ and $\overline{\mathbf{z}}$ are the actual displacement and virtual displacement fields of the structure, respectively, $\boldsymbol{Z}$ is the space of kinematically admissible virtual displacements, and $a_{\Omega}(\mathbf{z}, \overline{\mathbf{z}})$ and $\ell_{\Omega}(\overline{\mathbf{z}})$ are energy bilinear and load linear forms, respectively. The subscript $\Omega$ in Equation 5 is used to indicate the dependency of the governing equation on the shape of the structural domain.

The pointwise material derivative at $\mathbf{x} \in \Omega$ is defined as (Choi and Haug, 1983; Haug et al., 1986)

$$
\dot{z}=\lim _{\tau \rightarrow 0}\left[\frac{\mathbf{z}_{\tau}(\mathbf{x}+\tau \boldsymbol{V}(\mathbf{x}))-\mathbf{z}(\mathbf{x})}{\tau}\right] .
$$

If $\mathbf{z}_{\tau}$ has a regular extension to a neighborhood of $\Omega_{\tau}$, then

$$
\dot{z}(\mathbf{x})=\mathbf{z}^{\prime}(\mathbf{x})+\nabla \mathbf{z}^{T} \boldsymbol{V}(\mathbf{x}),
$$

where

$$
\mathbf{z}^{\prime}=\lim _{\tau \rightarrow 0}\left[\frac{\mathbf{z}_{\tau}(\mathbf{x})-\mathbf{z}(\mathbf{x})}{\tau}\right]
$$

is the partial derivative of $z$ and $\nabla=\left\{\partial / \partial x_{1}, \partial / \partial x_{2}, \partial / \partial x_{3}\right\}^{T}$ is the vector of gradient operators. One attractive feature of the partial derivative is that, given the smoothness assumption, it commutes with the derivatives with respect to $x_{i}, i=1,2$, and 3 , since they are derivatives with respect to independent variables, i.e.,

$$
\left(\frac{\partial \mathbf{z}}{\partial x_{i}}\right)^{\prime}=\frac{\partial}{\partial x_{i}}\left(\mathbf{z}^{\prime}\right), \quad i=1,2, \text { and } 3 .
$$

Let $\psi_{1}$ be a domain functional, defined as an integral over $\Omega_{\tau}$, i.e., 


$$
\psi_{1}=\int_{\Omega_{\tau}} f_{\tau}\left(\mathbf{x}_{\tau}\right) \mathrm{d} \Omega_{\tau}
$$

where $f_{\tau}$ is a regular function defined on $\Omega_{\tau}$. If $\Omega$ is $C^{k}$ regular, then the material derivative of $\psi_{1}$ at $\Omega$ is (Choi and Haug, 1983; Haug et al., 1986)

$$
\dot{\psi}_{1}=\int_{\Omega}\left[f^{\prime}(\mathbf{x})+\operatorname{div}(f(\mathbf{x}) \boldsymbol{V}(\mathbf{x}))\right] \mathrm{d} \Omega .
$$

For a functional form of

$$
\psi_{2}=\int_{\Omega_{\tau}} g\left(\mathbf{z}_{\tau}, \text { bolesymbol } \nabla \mathbf{z}_{\tau}\right) \mathrm{d} \Omega_{\tau},
$$

the material derivative of $\psi_{2}$ at $\Omega$ using Equations (9) and (11) is

$$
\dot{\psi}_{2}=\int_{\Omega}\left[g,_{z_{i}} \dot{\mathbf{z}}_{i}-\mathrm{g},{ }_{z_{i}}\left(\mathbf{z}_{i, j} V_{j}\right)+g,_{z_{i, j}} \dot{\mathrm{z}}_{i, j}-g,_{z_{i, j}}\left(z_{i, j} V_{j}\right)_{j}+\operatorname{div}(g \boldsymbol{V})\right] \mathrm{d} \Omega,
$$

for which, a comma is used to denote partial differentiation, e.g., $z_{i, j}=\partial z_{i} / \partial x_{j}, \dot{z}_{i, j}=$ $\partial \dot{z}_{i} / \partial x_{j}, g_{, z_{i}}=\partial g / \partial z_{i}, g_{, z_{i, j}}=\partial g / \partial z_{i, j}$ and $V_{j}$ is the $j$ th component of $\boldsymbol{V}$. In Equation (13), the material derivative $\dot{z}$ is the solution of the sensitivity equation obtained by taking the material derivative of Equation (5).

If no body force is involved, the variational equation (Equation (5)) can be written as

$$
a_{\Omega}(\mathbf{z}, \overline{\mathbf{z}}) \equiv \int_{\Omega} \sigma_{i j}(\mathbf{z}) \varepsilon_{i j}(\overline{\mathbf{z}}) \mathrm{d} \Omega=\ell_{\Omega}(\overline{\mathbf{z}}) \equiv \int_{\Gamma} T_{i} \bar{z}_{i} \mathrm{~d} \Gamma,
$$

where $\sigma_{i j}(\mathbf{z})$ and $\varepsilon_{i j}(\overline{\mathbf{z}})$ are the stress and strain tensors of the displacement $z$ and virtual displacement $\overline{\mathbf{z}}$, respectively, $T_{i}$ is the $i$ th component of the surface traction, and $\bar{z}_{i}$ is the $i$ th component of $\overline{\mathbf{z}}$. Taking the material derivative of both sides of Equation (14) and using Equation (9),

$$
a_{\Omega}(\dot{z}, \overline{\mathbf{z}})=\ell_{V}^{\prime}(\overline{\mathbf{z}})-a_{V}^{\prime}(\mathbf{z}, \overline{\mathbf{z}}), \quad \forall \overline{\mathbf{z}} \in \mathbf{Z},
$$

where the subscript $\boldsymbol{V}$ indicates the dependency of the terms on the velocity field. The terms $\ell_{V}^{\prime}(\overline{\mathbf{z}})$ and $a_{V}^{\prime}(\mathbf{z}, \overline{\mathbf{z}})$ can be further derived as (Choi and Haug, 1983; Haug et al., 1986)

$$
\ell_{V}^{\prime}(\overline{\mathbf{z}})=\int_{\Gamma}\left\{-T_{i}\left(z_{i, j} V_{j}\right)+\left[\left(T_{i} \bar{z}_{i}\right),{ }_{j} n_{j}+\kappa_{\gamma}\left(T_{i} \bar{z}_{i}\right)\right]\left(V_{i} n_{i}\right)\right\} \mathrm{d} \Gamma
$$

and

$$
a_{v}^{\prime}(\mathbf{z}, \overline{\mathbf{z}})=-\int_{\Omega}\left[\sigma_{i j}(\mathbf{z})\left(\bar{z}_{i, k} V_{k, j}\right)+\sigma_{i j}(\overline{\mathbf{z}})\left(z_{i, k} V_{k, j}\right)-\sigma_{i j}(\mathbf{z}) \varepsilon_{i j}(\overline{\mathbf{z}}) \operatorname{div} \boldsymbol{V}\right] \mathrm{d} \Omega,
$$


where $n_{i}$ is the $i$ th component of unit normal vector $\boldsymbol{n}, \kappa_{\Gamma}$ is the curvature of the boundary, $\bar{z}_{i, j}=\partial \bar{z}_{i} / \partial x_{j}$, and $V_{i, j}=\partial V_{i} / \partial x_{j}$.

To evaluate the sensitivity expression of Equation (13), a numerical method is needed to solve Equation (14); for which, a standard finite element method (FEM) was used in this study. If the solution $\mathbf{z}$ of Equation (14) is obtained using an FEM code, the same code can be used to solve Equation (15) for $\dot{z}$. This solution of $\dot{z}$ can be obtained efficiently since it requires only the evaluation of the same set of FEM matrix equations with a different fictitious load, which is the right hand side of Equation (15). The ABAQUS (Version 5.8) (ABAQUS, 1999) finite element code was used for all numerical calculations presented in forthcoming sections.

\section{The $J$-integral and its sensitivity}

\subsection{THE $J$-INTEGRAL}

Consider a body with a crack of length $a$, subject to mode-I loading. Using an arbitrary counter-clockwise path $\Gamma$ around the crack tip, as shown in Figure 2a, a formal definition of $J$ under mode-I condition is (Rice, 1968)

$$
J \stackrel{\text { def }}{=} \int_{\Gamma}\left(W n_{1}-T_{i} z_{i, 1}\right) \mathrm{d} \Gamma,
$$

where $W=\int \sigma_{i j} \mathrm{~d} \varepsilon_{i j}$ is the strain energy density with $\sigma_{i j}$ and $\varepsilon_{i j}$ representing components of stress and strain tensors, respectively, $z_{i}$ and $T_{i}=\sigma_{i j} n_{j}$ are the $i$ th components of the displacement and traction vectors, $\mathrm{d} \Gamma$ is the differential length along contour $\Gamma$, and $z_{i, 1}=$ $\partial z_{i} / \partial x_{1}$ is the differentiation of displacement $z_{i}$ with respect to $x_{1}$. The summation convention is adopted here for repeated indices.

Using the divergence theorem, the contour integral defined in Equation (18) can be expanded into an area integral in two dimensions, and volume integral in three dimensions, over a finite domain surrounding the crack tip or crack front. For two-dimensional problems involving linear- or nonlinear-elastic material under quasi-static conditions, in the absence of body forces, thermal strains, and crack-face tractions, Equation (18) reduces to (Shih et al., 1986; Moran and Shih, 1987; Li et al., 1985; Anderson, 1995)

$$
J=\int_{A}\left[\sigma_{i j} \frac{\partial z_{j}}{\partial x_{1}}-W \delta_{1 i}\right] \frac{\partial q}{\partial x_{i}} \mathrm{~d} A,
$$

where $\delta_{1 i}$ is the Kronecker delta, $q$ is an arbitrary but smooth weighting function equal to unity on $\Gamma_{0}$ and zero on $\Gamma_{1}$, and $A$ is the annular area enclosed by the inner contour $\Gamma_{0}$ and outer contour $\Gamma_{1}$, as shown in Figure 2b. In this study, the inner contour $\Gamma_{0}$ coincides with the crack tip. Hence, $A$ becomes the area inside the outer contour $\Gamma_{1}$. On further expansion,

$$
J=\int_{A}\left[\left(\sigma_{11} \frac{\partial z_{1}}{\partial x_{1}}+\sigma_{12} \frac{\partial z_{2}}{\partial x_{1}}\right) \frac{\partial q}{\partial x_{1}}+\left(\sigma_{21} \frac{\partial z_{1}}{\partial x_{1}}+\sigma_{22} \frac{\partial z_{2}}{\partial x_{1}}\right) \frac{\partial q}{\partial x_{2}}-W \frac{\partial q}{\partial x_{1}}\right] \mathrm{d} A
$$




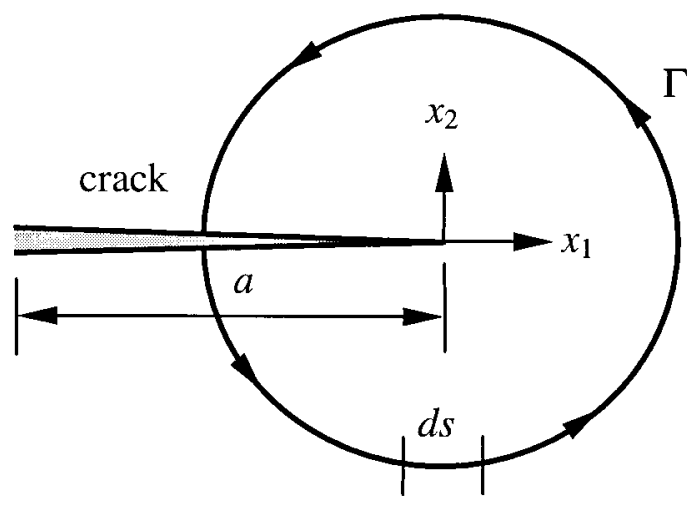

(a) Arbitrary contour around a crack tip

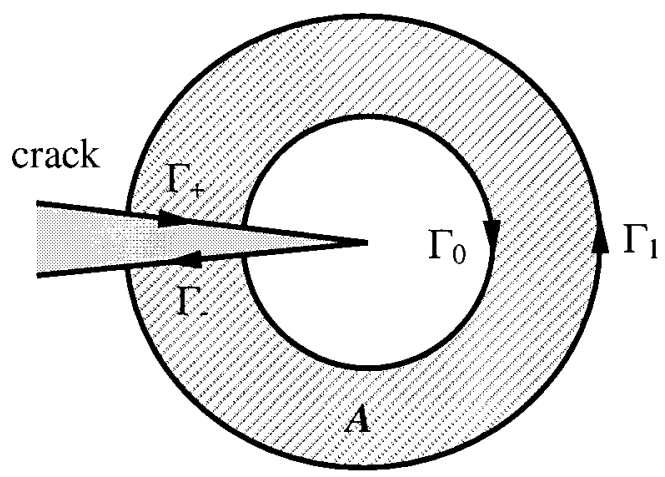

(b) Inner and outer contours enclosing $A$

Figure 2. $J$-integral fracture parameter; (a) arbitrary contour around a crack tip; (b) inner and outer contours enclosing $A$.

In Equation (20),

$$
\begin{aligned}
W & =\int_{0}^{\varepsilon_{i j}} \sigma_{i j} \mathrm{~d} \varepsilon_{i j} \\
& =\left\{\begin{array}{c}
\left(\frac{E}{1-v^{2}}\right)\left(\frac{\varepsilon_{11}^{2}}{2}+v \varepsilon_{11} \varepsilon_{22}\right)+\left(\frac{E}{1+v}\right) \varepsilon_{12}^{2}+\left(\frac{E}{1-v^{2}}\right)\left(\frac{\varepsilon_{22}^{2}}{2}+v \varepsilon_{11} \varepsilon_{22}\right), \\
\text { for plane stress } \\
\left(\frac{E}{(1-2 v)(1+v)}\right)\left(\frac{(1-v) \varepsilon_{11}^{2}}{2}+2 v \varepsilon_{11} \varepsilon_{22}+\frac{(1-v) \varepsilon_{22}^{2}}{2}\right)+\left(\frac{E}{1+v}\right) \varepsilon_{12}^{2}, \\
\text { for plane strain, }
\end{array}\right.
\end{aligned}
$$

where $E$ is the Young's modulus, $v$ is the Poisson's ratio for the material of the body, and $\varepsilon_{i j}$ is the strain field given by 


$$
\varepsilon_{i j}=\frac{1}{2}\left(\frac{\partial z_{i}}{\partial x_{j}}+\frac{\partial z_{j}}{\partial x_{i}}\right), \quad i, j=1,2
$$

\subsection{Sensitivity OF the $J$-INTEGRAL}

For two-dimensional plane stress or plane strain problems, once the stress-strain relationship is applied, Equation (20) can be expressed as

$$
J=\int_{A} h \mathrm{~d} A
$$

where

$$
h=h_{1}+h_{2}+h_{3}+h_{4}-h_{5}-h_{6} .
$$

The explicit expressions of $h, i=1, \ldots, 6$ are given in Appendix A for both plane stress and plane strain conditions. In relation to Equation (11), the material derivative of $J$-integral is

$$
\dot{J}=\int_{A}\left[h^{\prime}+\operatorname{div}(h \boldsymbol{V})\right] \mathrm{d} A
$$

where

$$
h^{\prime}=h_{1}^{\prime}+h_{2}^{\prime}+h_{3}^{\prime}+h_{4}^{\prime}-h_{5}^{\prime}-h_{6}^{\prime}
$$

and $\boldsymbol{V}=\left\{V_{1}, V_{2}\right\}^{T}$. Assuming the crack length $a$ to be the variable of interest, a change in crack length in the $x_{1}$ direction (mode I) only, i.e., $\boldsymbol{V}=\left\{V_{1}, 0\right\}^{T}$, results in the expression of Equation (25) as

$$
\dot{J}=\int_{A}\left(H_{1}+H_{2}+H_{3}+H_{4}-H_{5}-H_{6}\right) \mathrm{d} A,
$$

where

$$
H_{i}=h_{i}^{\prime}+\frac{\partial\left(h_{i} V_{1}\right)}{\partial x_{1}}, \quad i=1, \ldots, 6 .
$$

Equations (B1)-(B6) and (B7)-(B12) in Appendix B provide explicit expressions of $H_{i}, i=$ 1,6 , for plane stress and plane strain conditions, respectively. These expressions of $H_{i}, i=1,6$, when inserted in Equation (27), yield the first-order sensitivity of $J$ with respect to crack size. Note, when the velocity field is unity at the crack tip, $\dot{J}$ is equivalent to $\partial J / \partial a$.

The integral in Equation (27) is independent of the domain size $A$ and can be calculated numerically using standard Gaussian quadrature. A $2 \times 2$ or higher integration rule is recommended to calculate $\dot{J}$. A flow diagram for calculating the sensitivity of $J$ is shown in Figure 3 . 


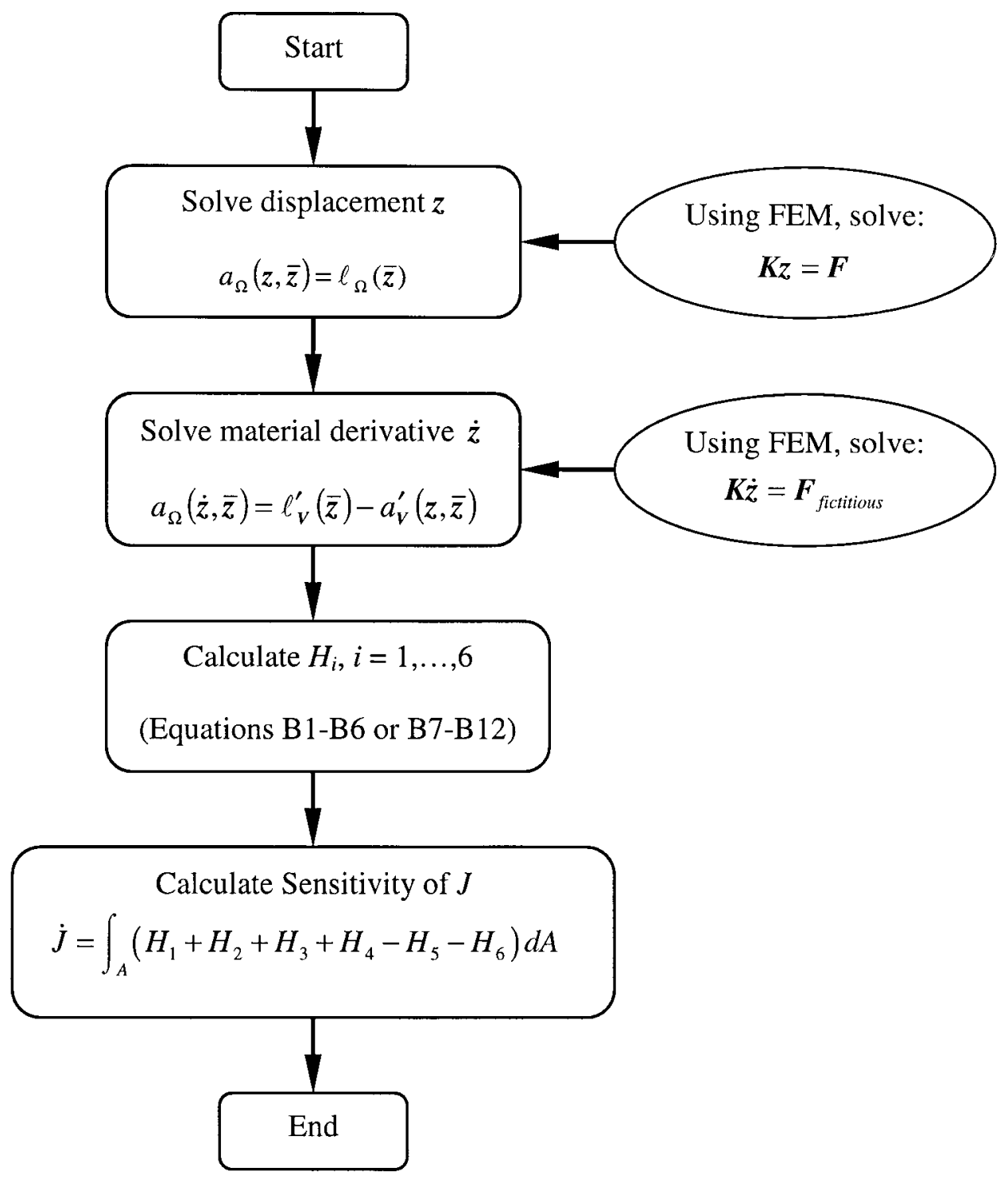

Figure 3. A flowchart for continuum sensitivity analysis of crack size.

\section{Probabilistic fracture mechanics and reliability}

\subsection{RANDOM PARAMETERS AND FRACTURE RESPONSE}

Consider a mode-I loaded linear-elastic cracked structure under uncertain mechanical and geometric characteristics subject to random loads. Denote by $\boldsymbol{X}$ an $N$-dimensional random vector with components $X_{1}, X_{2}, \cdots, X_{N}$ characterizing uncertainties in the load, crack geometry, and material properties. For example, if the crack size $a$, elastic modulus $E$, far-field applied stress magnitude $\sigma^{\infty}$, and mode-I fracture toughness at crack initiation $J_{I c}$, are modeled as input random variables, then $\mathbf{X}=\left\{a, E, \sigma^{\infty}, J_{I c}\right\}^{T}$. Let $J$ be a relevant crack-driving force that can be calculated using standard finite element analysis. Suppose the structure fails when $J>J_{I c}$. This requirement cannot be satisfied with certainty, since $J$ is dependent on the input vector 
$\boldsymbol{X}$ which is random, and $J_{I c}$ itself may be a random variable. Hence, the performance of the cracked structure should be evaluated by the reliability $P_{S}$, or its complement, the probability of failure $P_{F}\left(P_{S}=1-P_{F}\right)$, defined as

$$
P_{F} \stackrel{\text { def }}{=} \operatorname{Pr}[g(\mathbf{X})<0] \stackrel{\text { def }}{=} \int_{g(\mathbf{x})<0} f_{\mathbf{X}}(\mathbf{x}) \mathrm{d} \mathbf{x},
$$

where $f_{\mathbf{X}}(\mathbf{x})$ is the joint probability density function of $\boldsymbol{X}$, and

$$
g(\mathbf{x})=J_{I c}(\mathbf{x})-J(\mathbf{x})
$$

is the performance function. Note that $P_{F}$ in Equation (29) represents the probability of initiation of crack growth, which provides a conservative estimate of structural performance. A less conservative evaluation requires calculation of failure probability based on crack-instability criterion. The latter probability is more difficult to compute, since it must be obtained by incorporating crack-growth simulation in a finite element analysis. However, if suitable approximations of $J$ can be developed analytically, the crack instability-based failure probability can be easily calculated as well (Rahman, 1995).

\subsection{Reliability ANALYSis By FORM}

The generic expression of the failure probability in Equation (29) involves multi-dimensional probability integration for evaluation. In this study, the first-order reliability method (FORM) was used to compute this probability and is briefly described here to compute the probability of failure $P_{F}$ in Equation (29) assuming a generic $N$-dimensional random vector $\boldsymbol{X}$ and the performance function $g(\boldsymbol{x})$ defined by Equation (30).

The first-order reliability method is based on linear (first-order) approximation of the limit state surface $g(\boldsymbol{x})=0$ tangent to the closest point of the surface to the origin of the space. The determination of this point involves nonlinear constrained optimization and is usually performed in the standard Gaussian image of the original space. The FORM algorithm involves several steps. First, the space $\boldsymbol{x}$ of uncertain parameters $\boldsymbol{X}$ is transformed into a new $N$-dimensional space $\boldsymbol{u}$, consisting of independent standard Gaussian variables $\boldsymbol{U}$. The original limit state $g(\boldsymbol{x})=0$ is then mapped into the new limit state $g_{U}(\boldsymbol{u})=0$ in the $\boldsymbol{u}$ space. Second, the point on the limit state $g_{U}(\boldsymbol{u})=0$ having the shortest distance to the origin of the $\boldsymbol{u}$ space is determined using an appropriate nonlinear optimization algorithm. This point is referred to as the most probable point or the beta point, and has a distance $\beta_{H L}$ (known as reliability index) to the origin of the $\boldsymbol{u}$ space. Third, the limit state $g_{U}(\boldsymbol{u})=0$ is approximated by a hyperplane $g_{L}(\boldsymbol{u})=0$, tangent to it at the beta point. The probability of failure $P_{F}$ (Equation (29)) is thus approximated by $P_{F, 1}=\operatorname{Pr}\left[g_{L}(\boldsymbol{U})<0\right]$ in FORM and is given by (Madsen et al., 1986)

$$
P_{F, 1}=\Phi\left(-\beta_{H L}\right)
$$

where

$$
\Phi(u)=\frac{1}{\sqrt{2 \pi}} \int_{-\infty}^{u} \exp \left(-\frac{1}{2} \xi^{2}\right) d \xi
$$


is the cumulative probability distribution function of a standard Gaussian random variable.

A modified Hasofer-Lind-Rackwitz-Fiessler algorithm (Hasofer and Lind, 1974; Rackwitz and Fiessler, 1978; Liu and Kiureghian, 1991) was used to solve the associated optimization problem in this study. The first-order sensitivities were calculated analytically and are described in the following subsection.

\subsection{AnAlytical GRADients}

In $\boldsymbol{u}$ space, the objective function is quadratic; hence, calculation of its first-order derivative with respect to $u_{k}, k=1,2, \cdots, N$ is trivial. For the constraint function, i.e., the performance function, one must also calculate its derivative with respect to $u_{k}$. Assume that a transformation of $\mathbf{x} \in \mathfrak{R}^{N}$ to $\mathbf{u} \in \mathfrak{R}^{N}$, given by

$$
\mathbf{x}=\mathbf{x}(\mathbf{u}),
$$

exists. The performance function in the $\boldsymbol{u}$ space can then be expressed as

$$
g_{U}(\mathbf{u})=g(\mathbf{x}(\mathbf{u}))=J_{I c}(\mathbf{x}(\mathbf{u}))-J(\mathbf{x}(\mathbf{u})) .
$$

Using the chain rule of differentiation, the first-order derivative of $g_{U}(\mathbf{u})$ with respect to $u_{k}$ is

$$
\frac{\partial g_{U}(\mathbf{u})}{\partial u_{k}}=\sum_{j=1}^{N} \frac{\partial g}{\partial x_{j}} \frac{\partial x_{j}}{\partial u_{k}}=\sum_{j=1}^{M} \frac{\partial g}{\partial x_{j}} R_{j k},
$$

where $R_{j k}=\partial x_{j} / \partial u_{k}$ can be obtained from the explicit form of Equation (33). In LEFM with $\mathbf{X}=\left\{a, E, \sigma^{\infty}, J_{I c}\right\}^{T}$, the partial derivatives in the $\boldsymbol{x}$ space are

$$
\begin{gathered}
\frac{\partial g}{\partial a}=-\frac{\partial J}{\partial a} \\
\frac{\partial g}{\partial E}=-\frac{\partial J}{\partial E}=\frac{J}{E} \quad(\text { since } J \propto 1 / E), \\
\frac{\partial g}{\partial \sigma^{\infty}}=-\frac{\partial J}{\partial \sigma^{\infty}}=-\frac{2 J}{\sigma^{\infty}} \quad\left(\text { since } J \propto \sigma^{\infty 2}\right),
\end{gathered}
$$

and

$$
\frac{\partial g}{\partial J_{I c}}=1
$$

Using the proposed shape sensitivity method, the partial derivative of $J$ with respect to crack size can be easily calculated. Hence, for a given $\boldsymbol{u}$ or $\boldsymbol{x}$, all gradients of $g_{U}(\mathbf{u})$ can be evaluated analytically. Therefore, FORM or any other gradient-based reliability analysis can be performed efficiently. 


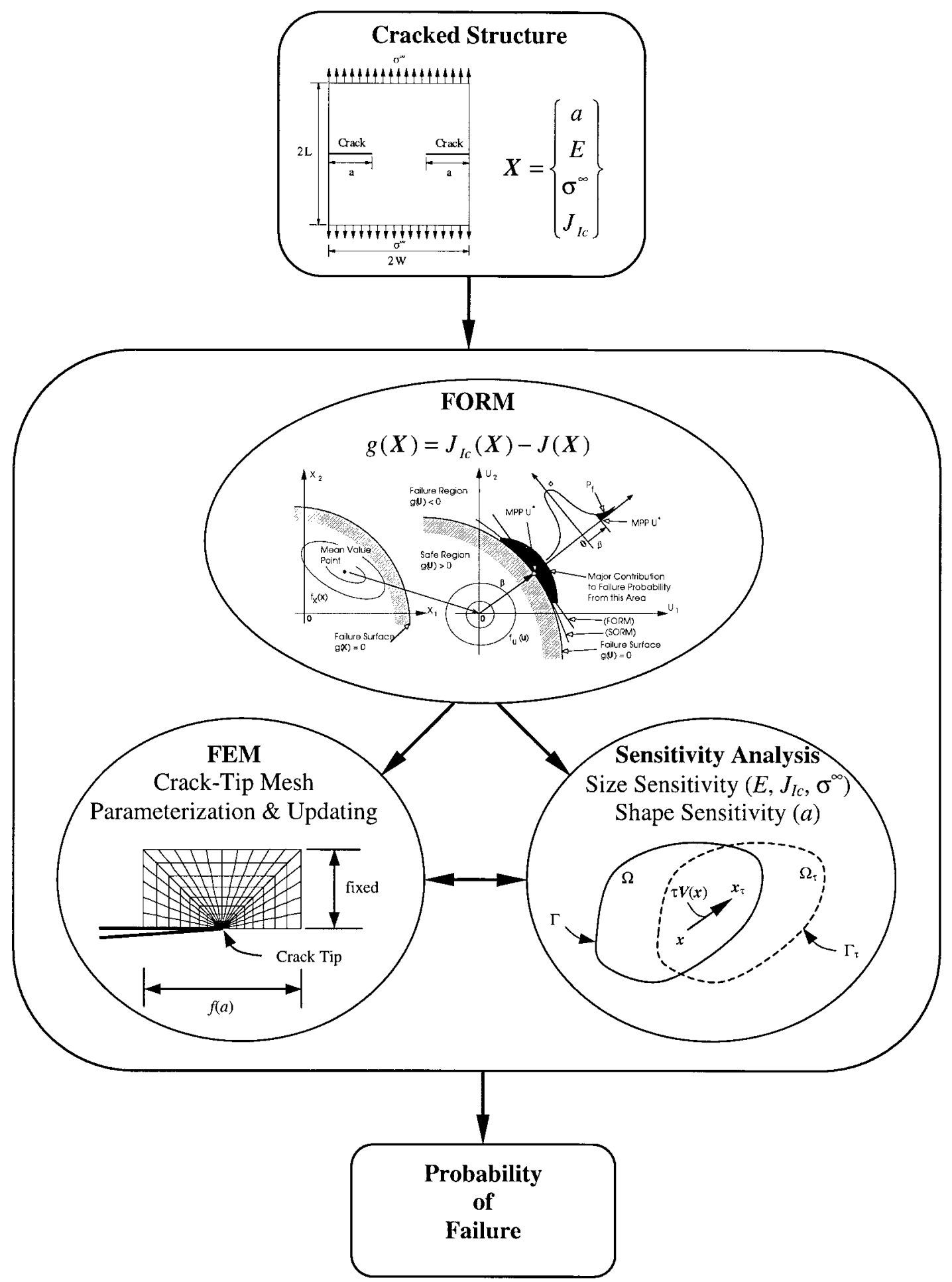

Figure 4. A flowchart for continuum sensitivity-based fracture reliability analysis. 


\subsection{INTERFACE}

Figure 4 illustrates a flowchart of the sensitivity-based FORM for fracture reliability analysis. In solving the optimization problem in FORM, one must be able to calculate $g(\boldsymbol{x})$ for a given $\boldsymbol{x}$. If an external code (e.g., commercial FEM code) is used for finite element analysis, an interface must be developed between the FEM and FORM modules. In addition, if the crack size is random, the crack-tip mesh must be parameterized with respect to crack size parameters, which can be achieved by appropriately modifying the FEM pre-processor module or randomizing the input files of FEM codes. Clearly, there is more than one way to perform such a parameterization. Nevertheless, the crack-tip mesh must be functionally dependent on the crack size such that the mesh quality remains adequate for any realization of crack size and the mapping from crack size to mesh movement is sufficiently smooth so that the performance function is differentiable. The gradients of $g(\boldsymbol{x})$ at any given mesh can be calculated using the sensitivity analysis module, as shown in Figure 4. For such calculations, the sensitivity analysis must also be connected with the external FEM code, as depicted in Figure 4.

\section{Numerical examples}

\subsection{EXAMPle 1: SENSITIVITY ANALYSiS OF M(T) SPECIMEN}

Consider a middle-tension panel $[\mathrm{M}(\mathrm{T})]$ with width $2 W=20$ units, length $2 L=20$ units and crack length $2 a$, subjected to a far-field remote tensile stress, $\sigma^{\infty}=1$ unit. Two crack sizes with normalized crack lengths $a / W=0.025$ and 0.05 were considered. The elastic modulus $E$ and Poisson's ratio $v$ were 26 units and 0.3 , respectively.

Figure 5a illustrates the geometry and loads of the $M(T)$ specimen. A finite element mesh for 1/4-model was used due to the double-symmetry of this $\mathrm{M}(\mathrm{T})$ problem, as shown in Figure 5b. Both plane stress and plane strain conditions were studied. Second-order elements from the ABAQUS (Version 5.8) (ABAQUS, 1999) element library were employed. For plane stress, the element type was CPS8R - the reduced integration, eight-noded quadrilateral element. The element type CPE8R was used for plane strain. The model consisted of 270 elements and 843 nodes. Focused elements with collapsed nodes were employed in the vicinity of crack tip. The domain (contour) of integration is depicted in Figure 5b. A $2 \times 2$ Gaussian integration was used.

Table 1 presents the numerical results for $J$ and $\partial J / \partial a$ for the $\mathrm{M}(\mathrm{T})$ problem. Both plane stress and plane strain conditions were analyzed. For each stress state, two sets of results are shown for $\partial J / \partial a$. The first set presents $\partial J / \partial a$ computed using the method described herein; the second set was calculated using the analytical solution for an infinite panel (Anderson, 1995). The results in Table 1 demonstrate that the continuum shape sensitivity analysis provides very accurate results for $\partial J / \partial a$ in comparison with the corresponding results from the analytical solution. Unlike the virtual crack extension technique, no mesh perturbation is required in the proposed method. The difference between the results of the proposed method and the analytical solution is less than $3 \%$.

\subsection{Example 2: Sensitivity analysis of SE(T) SPeCimen}

Consider a single-edged-tension [SE(T)] specimen with width $W=10$ units, length $L=10$ units, and crack length $a$, subjected to a far-field remote tensile stress $\sigma^{\infty}=1$ unit. Two 
$\sigma^{\infty}$

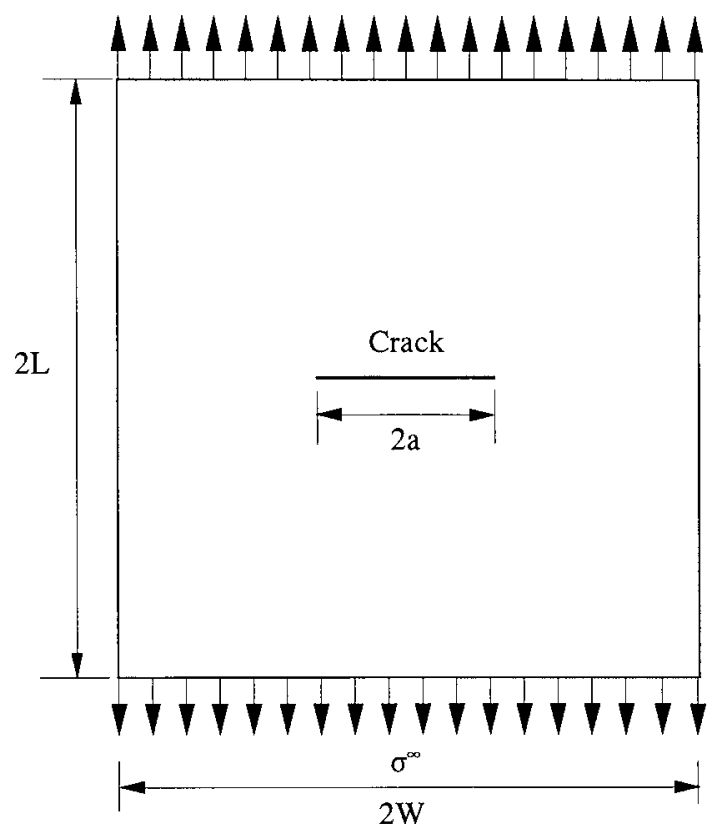

(a) Geometry and loads

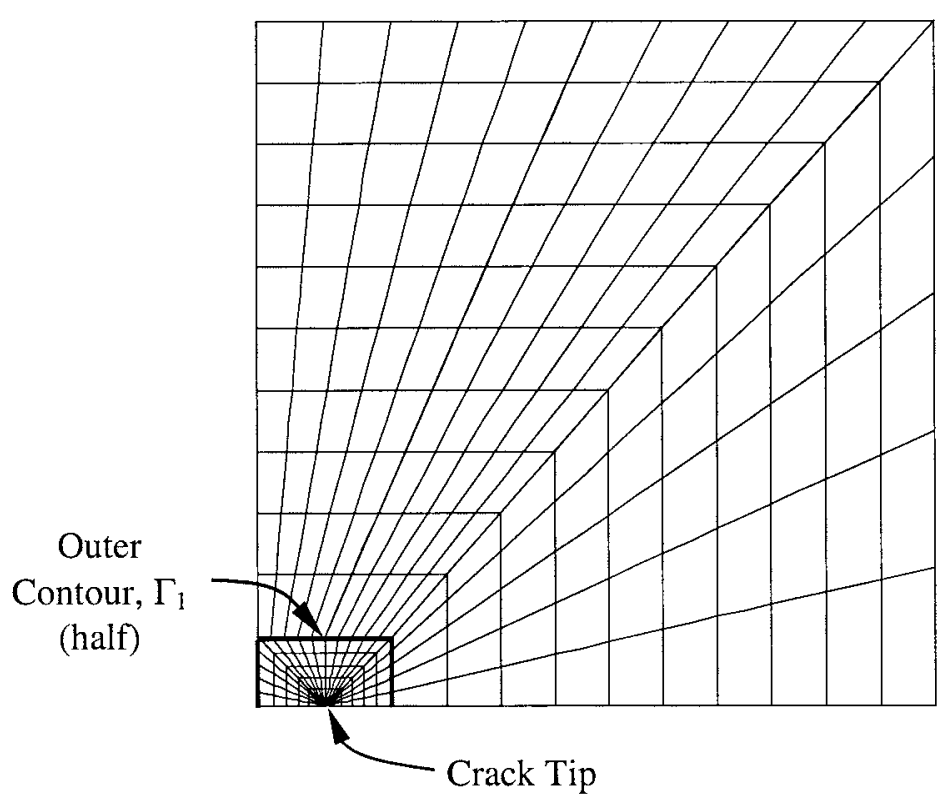

(b) Finite element mesh (1/4 model)

Figure 5. M(T) specimen under mode-I loading; (a) geometry and loads; (b) finite element mesh (1/4 model). 
Table 1. Sensitivity of $J$ for $\mathrm{M}(\mathrm{T})$ specimen by the proposed and analytical methods

\begin{tabular}{|c|c|c|c|c|}
\hline \multirow[t]{2}{*}{$a / W$} & \multirow[t]{2}{*}{$J$-integral } & \multicolumn{2}{|c|}{ Sensitivity of $J$-integral $(\partial J / \partial a)$} & \multirow[b]{2}{*}{$\begin{array}{l}\text { Difference } \\
(\%)\end{array}$} \\
\hline & & $\begin{array}{l}\text { Proposed } \\
\text { method }\end{array}$ & $\begin{array}{l}\text { Analytical } \\
\text { method }\end{array}$ & \\
\hline
\end{tabular}

(a) Plane stress

$\begin{array}{llllr}0.025 & 3.04 \times 10^{-5} & 1.22 \times 10^{-4} & 1.21 \times 10^{-4} & -0.83 \\ 0.05 & 6.01 \times 10^{-5} & 1.19 \times 10^{-4} & 1.21 \times 10^{-4} & 1.65\end{array}$

(b) Plane strain

\begin{tabular}{lllll}
0.025 & $2.77 \times 10^{-5}$ & $1.12 \times 10^{-4}$ & $1.10 \times 10^{-4}$ & -1.82 \\
0.05 & $5.48 \times 10^{-5}$ & $1.13 \times 10^{-4}$ & $1.10 \times 10^{-4}$ & -2.72 \\
\hline
\end{tabular}

$\overline{{ }^{a} \text { Difference }=(\partial J / \partial a \text { by analytical method }-\partial J / \partial a \text { by proposed }}$ method $) \times 100 / \partial J / \partial a$ by analytical method.

Table 2. Sensitivity of $J$ for $\mathrm{SE}(\mathrm{T})$ specimen by the proposed and finite-difference methods

\begin{tabular}{lllll}
\hline$a / W$ & $J$-integral & \multicolumn{2}{c}{ Sensitivity of $J$-integral $(\partial J / \partial a)$} & \\
\cline { 3 - 4 } & & $\begin{array}{l}\text { Proposed } \\
\text { method }\end{array}$ & $\begin{array}{l}\text { Finite } \\
\text { difference }\end{array}$ & $(\%)$ \\
\hline \multicolumn{5}{c}{ (a) Plane stress } \\
0.25 & $5.80 \times 10^{-7}$ & $4.63 \times 10^{-7}$ & $4.59 \times 10^{-7}$ & -0.87 \\
0.5 & $4.70 \times 10^{-6}$ & $3.48 \times 10^{-7}$ & $3.54 \times 10^{-7}$ & 1.69 \\
& & (b) Plane strain & \\
0.25 & $5.44 \times 10^{-7}$ & $4.28 \times 10^{-7}$ & $4.30 \times 10^{-7}$ & 0.65 \\
0.5 & $4.39 \times 10^{-6}$ & $3.52 \times 10^{-7}$ & $3.49 \times 10^{-7}$ & -0.86 \\
\hline
\end{tabular}

${ }^{\mathrm{a} D i f f e r e n c e}=(\partial J / \partial a$ by finite difference method $-\partial J / \partial a$ by proposed method $) \times 100 / \partial J / \partial a$ by finite difference method.

crack sizes with normalized crack lengths $a / W=0.25$ and 0.5 were considered. The elastic modulus $E$ and Poisson's ratio $v$ were 30 units and $v=0.25$, respectively.

The geometry and loads of the SE(T) specimen are shown in Figure 6a. Due to singlesymmetry of the $\mathrm{SE}(\mathrm{T})$ problem, a finite element mesh for 1/2-model was used, as shown in Figure $6 \mathrm{~b}$. The model consisted of 280 elements and 873 nodes. As before, both plane stress and plane strain conditions were studied.

Table 2 presents the numerical results for $J$ and $\partial J / \partial a$ for the SE(T) problem. Two sets of results are shown for $\partial J / \partial a$, the first computed using the proposed method and second calculated using the finite-difference method, since no analytical solution was available for this problem. A one-percent perturbation of crack length was used in the finite-difference calculations. As in Example 1, the results in Table 2 also demonstrate that continuum shape sensitivity analysis provides accurate estimates of $\partial J / \partial a$ as compared with corresponding results from the finite-difference method. Unlike the virtual crack extension technique, no 


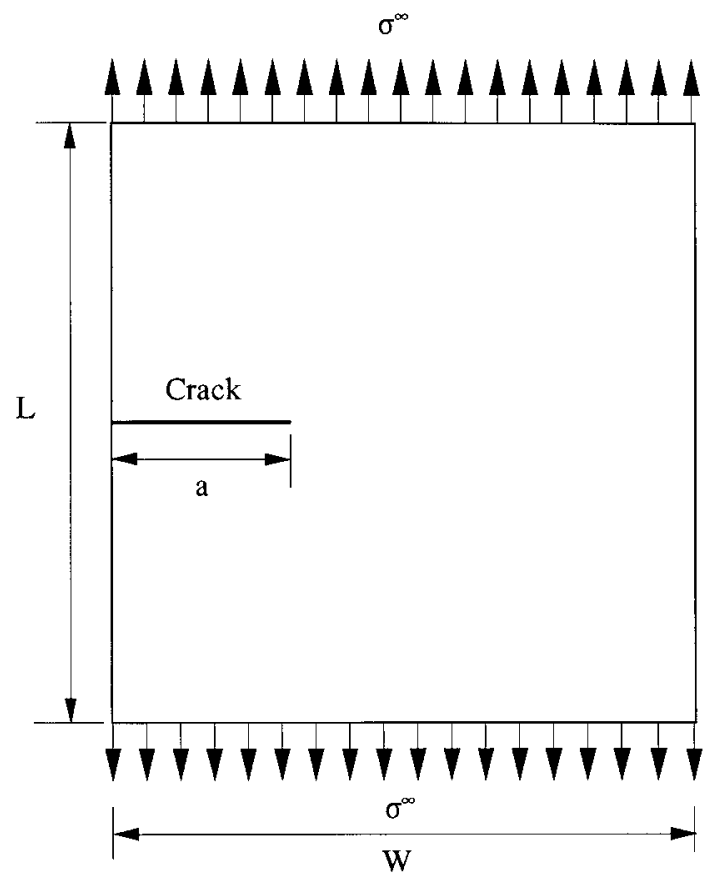

(a) Geometry and loads

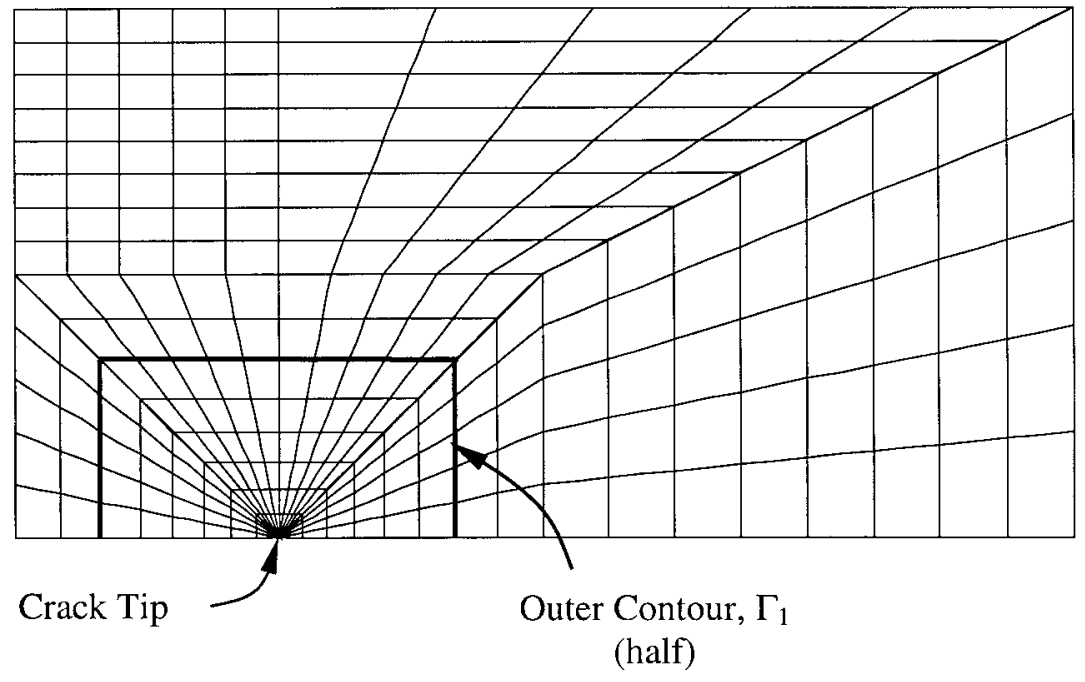

(b) Finite element mesh (1/2 model)

Figure 6. SE(T) specimen under mode-I loading; (a) geometry and loads; (b) finite element mesh (1/2 model). 


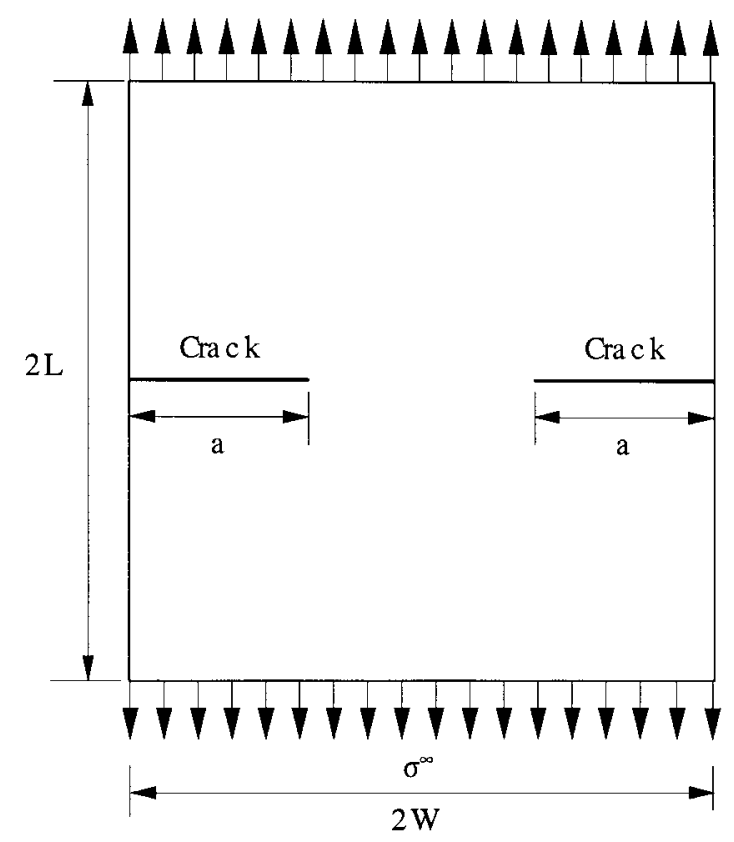

(a) Geometry and loads

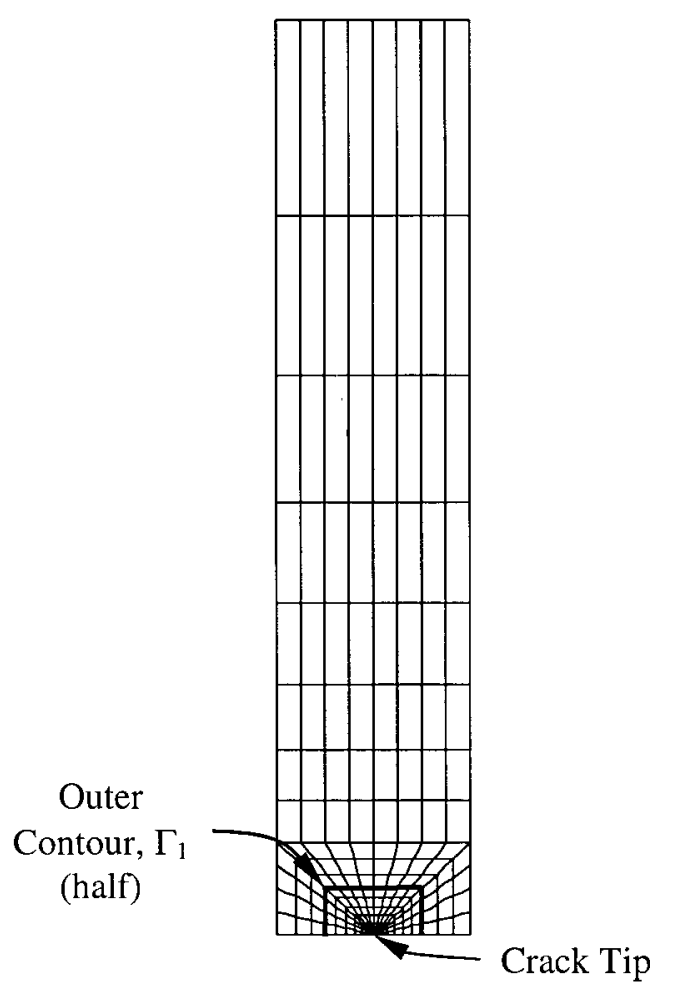

(b) Finite element mesh (1/4 model)

Figure 7. DE(T) specimen under mode-I loading; (a) geometry and loads; (b) finite element mesh (1/4 model).

mesh perturbation is required using the proposed method. The difference between the results of the proposed method and the finite-difference method is less than $2 \%$.

\subsection{EXAMPLE 3: Reliability ANALYSIS OF DE(T) SPECIMEN}

Consider a double-edged-notched tension [DE(T)] specimen (Figure 7a) with width $2 W=$ $1.016 \mathrm{~m}$ (40 inches), length $2 L=5.08 \mathrm{~m}$ (200 inches), and crack length $a$, subject to a far-field tensile stress $\sigma^{\infty}$, as shown in Figure 7a. The load, crack size, and material properties were treated as statistically independent random variables. Table 3 presents the mean, coefficient of variation $(\mathrm{COV})$, and probability distribution for each of these random parameters. Some of these values in Table 3 came from statistical characterization of actual material property data (Rahman et al., 1995). The Poisson's ratio of $v=0.3$ was assumed to be deterministic.

Due to the double-symmetry of this DE(T) problem, Figure $7 \mathrm{~b}$ shows a finite element mesh (at mean crack length) of a 1/4-model. A total of 208 elements and 691 nodes were used in the mesh. Second-order elements from ABAQUS element library were used. Both plane stress and plane strain conditions were studied. The element types are the same as in Example 1. Focused elements were used in the vicinity of crack tip. The domain (contour) of integration is indicated in Figure 7b. A $2 \times 2$ Gaussian integration was used.

Using continuum sensitivity analysis of $J$, a number of probabilistic analyses were performed to calculate the probability of failure $P_{F}{ }^{1}$ of the $\mathrm{DE}(\mathrm{T})$ specimen, as a function of

\footnotetext{
${ }^{1}$ The failure probability in this paper refers to probability of fracture initiation (see Equations (29) and (30)).
} 
Guofeng Chen et al.

Table 3. Statistical properties of random input for $\mathrm{DE}(\mathrm{T})$ specimen

\begin{tabular}{lclll}
\hline Random variable & Mean & $\mathrm{COV}^{\mathrm{a}}$ & $\begin{array}{l}\text { Probability } \\
\text { distribution }\end{array}$ & Reference \\
\hline Normalized Crack Length $(a / W)$ & 0.5 & Variable $^{\mathrm{b}}$ & Lognormal & $\mathbf{-}^{\mathrm{c}}$ \\
Elastic modulus $(E)$ & $206.8 \mathrm{GPa}$ & 0.05 & Gaussian & $\mathbf{-}^{\mathrm{c}}$ \\
Initiation fracture toughness $\left(J_{I c}\right)$ & $1242.6 \mathrm{~kJ} / \mathrm{m}^{2}$ & 0.47 & Lognormal & Pahman et al (1995) \\
Far-field tensile stress $\left(\sigma^{\infty}\right)$ & Variable $^{\mathrm{b}}$ & 0.1 & Gaussian & - $^{\mathrm{c}}$ \\
\hline
\end{tabular}

${ }^{\mathrm{a} C o e f f i c i e n t}$ of variation $(\mathrm{COV})=$ standard deviation/mean.

$\mathrm{b}$ Arbitrarily varied.

${ }^{\mathrm{c}}$ Arbitrarily assumed.

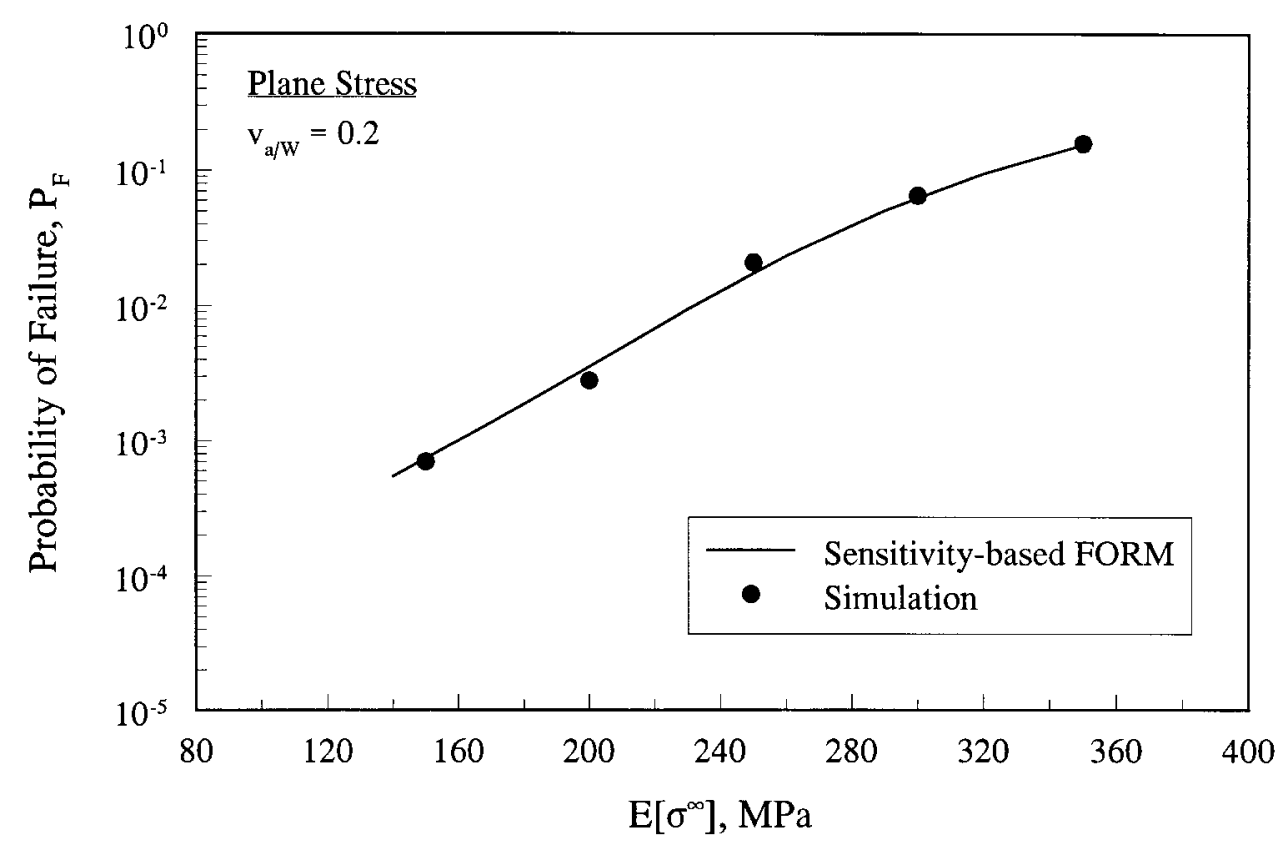

Figure 8. Failure probability of $\mathrm{DE}(\mathrm{T})$ specimen by FORM and simulation.

mean far-field tensile stress $E\left[\sigma^{\infty}\right]$, where $\mathrm{E}[\cdot]$ is the expectation (mean) operator. Figure 8 plots the $P_{F}$ vs. $E\left[\sigma^{\infty}\right]$ results for $v_{a / W}=20$ percent and the plane stress condition, where $v_{a / W}$ is the COV of the normalized crack length $a / W$. The probability of failure was calculated using sensitivity-based FORM and Monte Carlo simulation. For the Monte Carlo simulation, the sample size varied and was at least 10 times the inverse of the estimated failure probability. As can be seen in Figure 8, the probability of failure by FORM is in good agreement with the simulation results.

Figures $9 \mathrm{a}$ and $9 \mathrm{~b}$ show the plots of $P_{F}$ vs. $E\left[\sigma^{\infty}\right]$ using FORM for plane stress and plane strain conditions, respectively, for both deterministic $\left(v_{a / W}=0\right)$ and random $\left(v_{a / W}=10\right.$, 20 , and 40 percent) crack sizes. As expected, the results indicate that the failure probability increases with the COV (uncertainty) of $a / W$, and can be much larger than the probabilities calculated for a deterministic crack size, particularly when the uncertainty of $a / W$ is large. The probability of failure in plane stress is slightly larger than that in plane strain, regardless of the load intensity, since $J$ in plane stress is $\left(1-v^{2}\right)^{-1}$ times larger than $J$ in plane strain. 


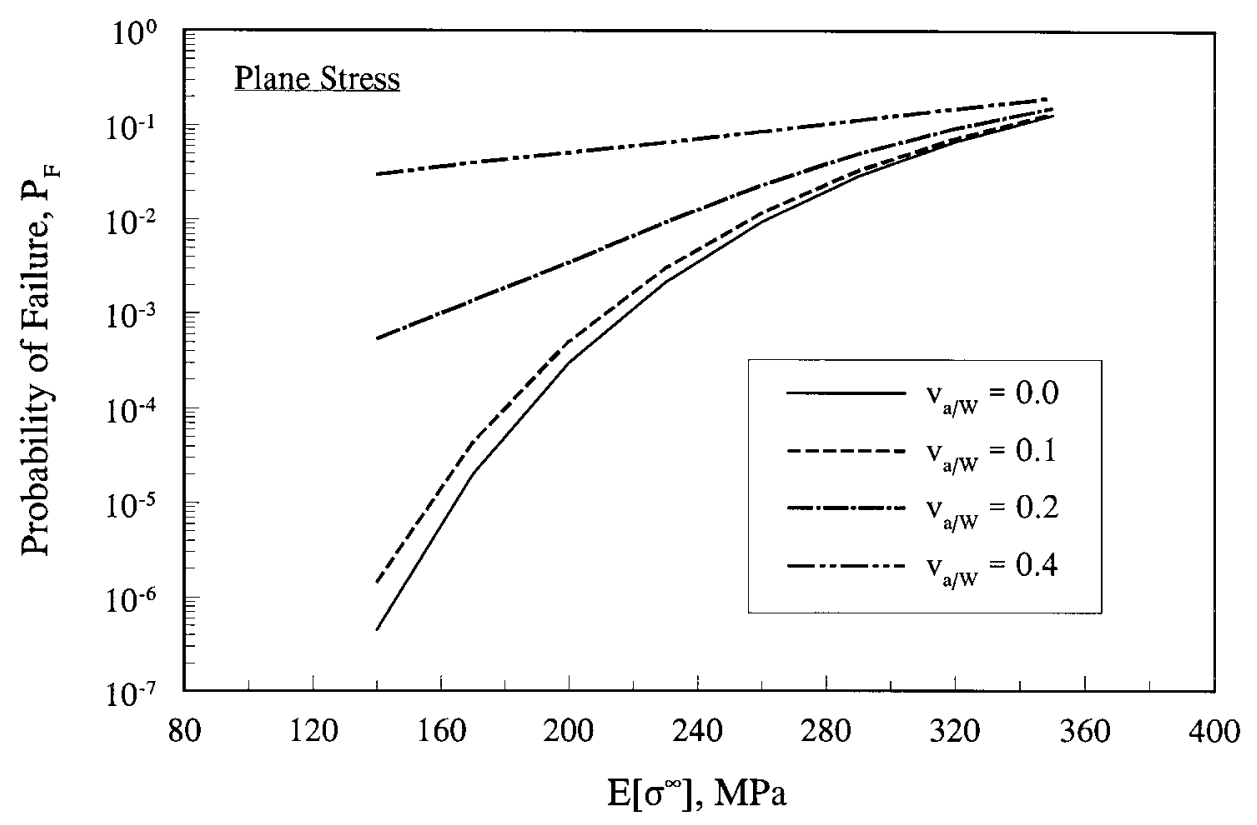

(a) plane stress

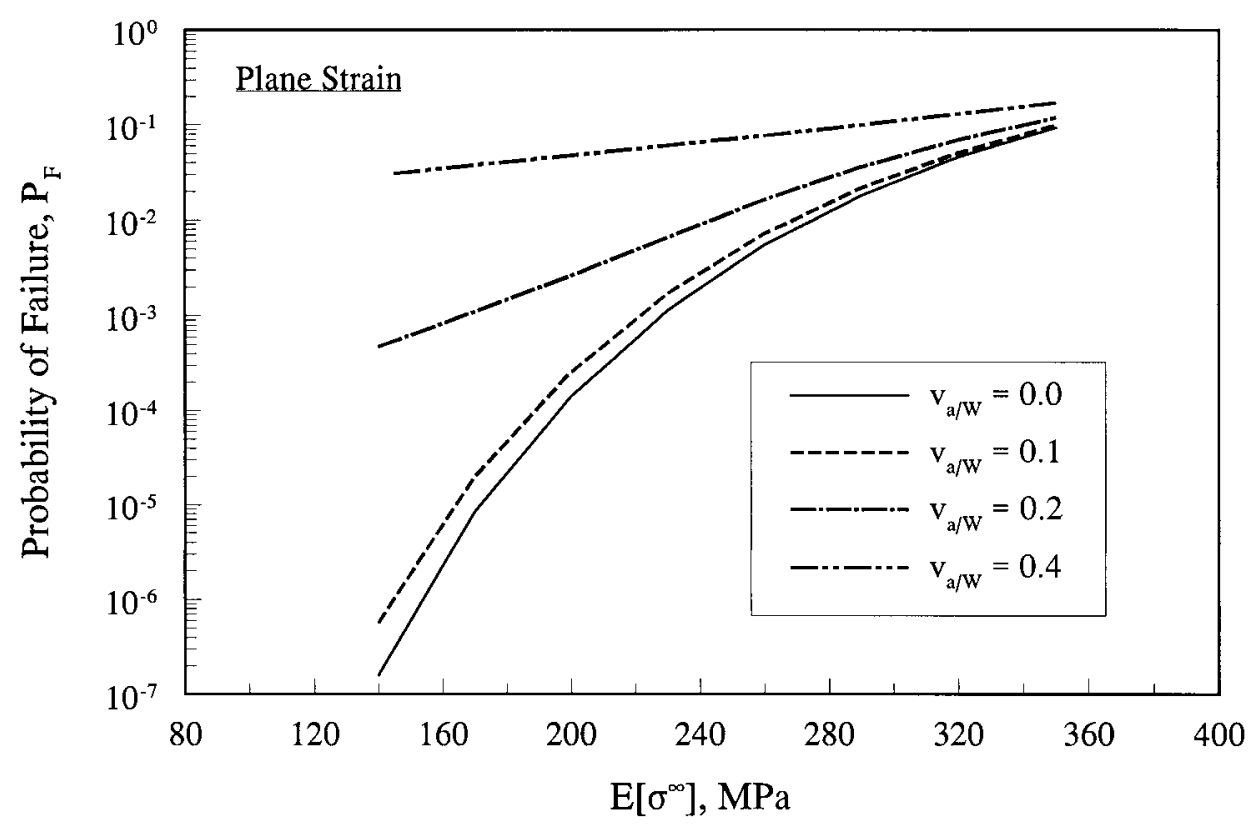

(b) plane strain

Figure 9. Failure probability of DE(T) specimen by FORM for various uncertainties in crack size; (a) plane stress; (b) plane strain. 
However, the difference in the resultant failure probabilities is small for the value of $v=0.3$ used. Furthermore, the fracture toughness $\left(J_{I c}\right)$ was assumed to be the same for both the plane stress and plane strain conditions in these calculations. Typically, however, the toughness for plane stress is higher than for plane strain. Due to a lack of data, this issue was not investigated in this study; hence, any relative comparison of failure probabilities in plane stress and plane strain should be made with caution. Note, all failure probabilities were calculated using the proposed sensitivity analysis, as represented by the flowcharts in Figures 3 and 4.

\section{Summary and conclusions}

A new method was developed for shape sensitivity analysis of a crack in a homogeneous, isotropic, and linear-elastic body subject to mode-I loading. The method involves the material derivative concept of continuum mechanics, domain integral representation of the $J$-integral, and direct differentiation. Unlike virtual crack extension techniques, no mesh perturbation is required in the proposed method. Since the governing variational equation is differentiated prior to the process of discretization, the resulting sensitivity equations are independent of any approximate numerical techniques, such as the finite element method, boundary element method, or others. Existing methods based on the expression of $J$ as a rate of potential energy require second-order sensitivity of potential energy to yield the first-order sensitivity of $J$. Since the proposed method requires only first-order sensitivities of stress and strain, it is much simpler and more efficient than existing methods. Numerical results demonstrate that the maximum difference in calculating the sensitivity of $J$ using the developed method is less than three percent, compared with the analytical solution or finite-difference results. Based on continuum sensitivities, the first-order reliability method was formulated to conduct probabilistic fracture-mechanics analysis. A numerical example has been presented to demonstrate the effectiveness of the proposed sensitivity equations for probabilistic analysis. Since all gradients are calculated analytically, the reliability analysis of cracks can be performed efficiently.

\section{Acknowledgements}

This research was supported by the Faculty Early Career Development Award (second author) of the U.S. National Science Foundation (Grant No. CMS-9733058). The NSF program directors were Drs. Sunil Saigal and Ken Chong.

\section{Appendix A. The $h$-functions}

For plane stress,

$$
\begin{gathered}
h_{1}=\frac{E}{1-v^{2}} \frac{\varepsilon_{11}^{2}}{2} \frac{\partial q}{\partial x_{1}}, \\
h_{2}=\frac{E}{1+v} \varepsilon_{12} \frac{\partial z_{2}}{\partial x_{1}} \frac{\partial q}{\partial x_{1}},
\end{gathered}
$$




$$
\begin{gathered}
h_{3}=\frac{E}{1+v} \varepsilon_{12} \varepsilon_{11} \frac{\partial q}{\partial x_{2}}, \\
h_{4}=\frac{E}{1-v^{2}}\left(\varepsilon_{22}+v \varepsilon_{11}\right) \frac{\partial z_{2}}{\partial x_{1}} \frac{\partial q}{\partial x_{2}}, \\
h_{5}=\frac{E}{1+v} \varepsilon_{12}^{2} \frac{\partial q}{\partial x_{1}},
\end{gathered}
$$

and

$$
\left.h_{6}=\frac{E}{1-v^{2}}\left(\frac{\varepsilon_{22}^{2}}{2}+v \varepsilon_{11} \varepsilon_{22}\right)\right) \frac{\partial q}{\partial x_{1}} .
$$

For plane strain,

$$
\begin{gathered}
h_{1}=\frac{E(1-v)}{(1+v)(1-2 v)} \frac{\varepsilon_{11}^{2}}{2} \frac{\partial q}{\partial x_{1}}, \\
h_{2}=\frac{E}{1+v} \varepsilon_{12} \frac{\partial z_{2}}{\partial x_{1}} \frac{\partial q}{\partial x_{1}}, \\
h_{3}=\frac{E}{1+v} \varepsilon_{12} \varepsilon_{11} \frac{\partial q}{\partial x_{2}}, \\
h_{4}=\frac{E}{(1+v)(1-2 v)}\left[(1-v) \varepsilon_{22}+v \varepsilon_{11}\right] \frac{\partial z_{2}}{\partial x_{1}} \frac{\partial q}{\partial x_{2}}, \\
h_{5}=\frac{E}{1+v} \varepsilon_{12}^{2} \frac{\partial q}{\partial x_{1}},
\end{gathered}
$$

and

$$
h_{6}=\frac{E}{(1+v)(1-2 v)}\left[\frac{\varepsilon_{22}^{2}}{2}(1-v)+v \varepsilon_{11} \varepsilon_{22}\right] \frac{\partial q}{\partial x_{1}} .
$$

\section{Appendix B. The H-functions}

For plane stress, 


$$
\begin{aligned}
H_{1}=\frac{E}{1-v^{2}} \frac{\partial q}{\partial x_{1}}\left[\varepsilon_{11} \frac{\partial \dot{z}_{1}}{\partial x_{1}}-\varepsilon_{11}^{2} \frac{\partial V_{1}}{\partial x_{1}}\right], \\
H_{2}=\frac{E}{1+v} \frac{\partial q}{\partial x_{1}}\left[\frac{\partial z_{2}}{\partial x_{1}}\left(\frac{\partial \dot{z}_{2}}{\partial x_{1}}+\frac{1}{2} \frac{\partial \dot{z}_{1}}{\partial x_{2}}-\frac{1}{2} \varepsilon_{11} \frac{\partial V_{1}}{\partial x_{2}}-\frac{\partial z_{2}}{\partial x_{1}} \frac{\partial V_{1}}{\partial x_{1}}\right)\right. \\
\left.+\frac{1}{2} \frac{\partial z_{1}}{\partial x_{2}}\left(\frac{\partial \dot{z}_{2}}{\partial x_{1}}-\frac{\partial z_{2}}{\partial x_{1}} \frac{\partial V_{1}}{\partial x_{1}}\right)\right], \\
H_{3}=\frac{1}{2} \frac{E}{1+v} \frac{\partial q}{\partial x_{2}}\left[\varepsilon_{11}\left(\frac{\partial \dot{z}_{2}}{\partial x_{1}}+\frac{\partial \dot{z}_{1}}{\partial x_{2}}\right)+2 \varepsilon_{12} \frac{\partial \dot{z}_{1}}{\partial x_{1}}-\varepsilon_{11}^{2} \frac{\partial V_{1}}{\partial x_{2}}-\varepsilon_{11} \frac{\partial z_{2}}{\partial x_{1}} \frac{\partial V_{1}}{\partial x_{1}}\right] \\
-\varepsilon_{11} \varepsilon_{12} \frac{E}{1+v} \frac{\partial q}{\partial x_{1}} \frac{\partial V_{1}}{\partial x_{2}}, \\
H_{4}=\frac{E}{1-v^{2}} \frac{\partial q}{\partial x_{2}} \frac{\partial z_{2}}{\partial x_{1}}\left(\frac{\partial \dot{z}_{2}}{\partial x_{2}}+v \frac{\partial \dot{z}_{1}}{\partial x_{1}}\right)-\frac{E}{1-v^{2}} \frac{\partial q}{\partial x_{2}}\left[\left(\frac{\partial z_{2}}{\partial x_{1}}\right)^{2} \frac{\partial V_{1}}{\partial x_{2}}\right. \\
\left.+v \varepsilon_{11} \frac{\partial z_{2}}{\partial x_{1}} \frac{\partial V_{1}}{\partial x_{1}}\right]+\frac{E}{1-v^{2}}\left(\varepsilon_{22}+v \varepsilon_{11}\right)\left[\frac{\partial q}{\partial x_{2}} \frac{\partial \dot{z}_{2}}{\partial x_{1}}-\frac{\partial q}{\partial x_{1}} \frac{\partial z_{2}}{\partial x_{1}} \frac{\partial V_{1}}{\partial x_{2}}\right], \\
H_{5}=\frac{E}{1+v} \frac{\partial q}{\partial x_{1}} \varepsilon_{12}\left[\frac{\partial \dot{z}_{1}}{\partial x_{2}}+\frac{\partial \dot{z}_{2}}{\partial x_{1}}-\frac{\partial z_{2}}{\partial x_{1}} \frac{\partial V_{1}}{\partial x_{1}}-\varepsilon_{11} \frac{\partial V_{1}}{\partial x_{2}}\right],
\end{aligned}
$$

and

$$
H_{6}=\frac{E}{1-v^{2}} \frac{\partial q}{\partial x_{1}}\left[\left(\varepsilon_{22}+v \varepsilon_{11}\right)\left(\frac{\partial \dot{z}_{2}}{\partial x_{2}}-\frac{\partial z_{2}}{\partial x_{1}} \frac{\partial V_{1}}{\partial x_{2}}\right)+v \varepsilon_{22}\left(\frac{\partial \dot{z}_{1}}{\partial x_{1}}-\varepsilon_{11} \frac{\partial V_{1}}{\partial x_{1}}\right)\right]
$$

For plane strain,

$$
\begin{gathered}
H_{1}=\frac{E(1-v)}{(1-2 v)(1+v)} \frac{\partial q}{\partial x_{1}}\left[\varepsilon_{11} \frac{\partial \dot{z}_{1}}{\partial x_{1}}-\varepsilon_{11}^{2} \frac{\partial V_{1}}{\partial x_{1}}\right], \\
H_{2}=\frac{E}{1+v} \frac{\partial q}{\partial x_{1}}\left[\frac{\partial z_{2}}{\partial x_{1}}\left(\frac{\partial \dot{z}_{2}}{\partial x_{1}}+\frac{1}{2} \frac{\partial \dot{z}_{1}}{\partial x_{2}}-\frac{1}{2} \varepsilon_{11} \frac{\partial V_{1}}{\partial x_{2}}-\frac{\partial z_{2}}{\partial x_{1}} \frac{\partial V_{1}}{\partial x_{1}}\right)\right. \\
\left.+\frac{1}{2} \frac{\partial z_{1}}{\partial x_{2}}\left(\frac{\partial \dot{z}_{2}}{\partial x_{1}}-\frac{\partial z_{2}}{\partial x_{1}} \frac{\partial V_{1}}{\partial x_{1}}\right)\right]
\end{gathered}
$$




$$
\begin{aligned}
& H_{3}= \frac{1}{2} \frac{E}{1+v} \frac{\partial q}{\partial x_{2}}\left[\varepsilon_{11}\left(\frac{\partial \dot{z}_{2}}{\partial x_{1}}+\frac{\partial \dot{z}_{1}}{\partial x_{2}}\right)+2 \varepsilon_{12} \frac{\partial \dot{z}_{1}}{\partial x_{1}}-\varepsilon_{11}^{2} \frac{\partial V_{1}}{\partial x_{2}}-\varepsilon_{11} \frac{\partial z_{2}}{\partial x_{1}} \frac{\partial V_{1}}{\partial x_{1}}\right] \\
&-\varepsilon_{11} \varepsilon_{12} \frac{E}{1+v} \frac{\partial q}{\partial x_{1}} \frac{\partial V_{1}}{\partial x_{2}} \\
& H_{4}= \frac{E}{(1-2 v)(1+v)} \frac{\partial q}{\partial x_{2}} \frac{\partial z_{2}}{\partial x_{1}}\left[v\left(\frac{\partial \dot{z}_{1}}{\partial x_{1}}-\varepsilon_{11} \frac{\partial V_{1}}{\partial x_{1}}\right)\right. \\
&\left.+(1-v)\left(\frac{\partial \dot{z}_{2}}{\partial x_{2}}-\frac{\partial z_{2}}{\partial x_{1}} \frac{\partial V_{1}}{\partial x_{2}}\right)\right] \\
&+\frac{E}{(1-2 v)(1+v)}\left[(1-v) \varepsilon_{22}+v \varepsilon_{11}\right]\left[\frac{\partial q}{\partial x_{2}} \frac{\partial \dot{z}_{2}}{\partial x_{1}}-\frac{\partial z_{2}}{\partial x_{1}} \frac{\partial q}{\partial x_{1}} \frac{\partial V_{1}}{\partial x_{2}}\right], \\
& H_{5}=\frac{E}{1+v} \frac{\partial q}{\partial x_{1}} \varepsilon_{12}\left[\frac{\partial \dot{z}_{1}}{\partial x_{2}}+\frac{\partial \dot{z}_{2}}{\partial x_{1}}-\varepsilon_{11} \frac{\partial V_{1}}{\partial x_{2}}-\frac{\partial z_{2}}{\partial x_{1}} \frac{\partial V_{1}}{\partial x_{1}}\right],
\end{aligned}
$$

and

$$
\begin{aligned}
H_{6}= & \frac{E}{(1-2 v)(1+v)} \frac{\partial q}{\partial x_{1}}\left[\left((1-v) \varepsilon_{22}+v \varepsilon_{11}\right)\left(\frac{\partial \dot{z}_{2}}{\partial x_{2}}-\frac{\partial z_{2}}{\partial x_{1}} \frac{\partial V_{1}}{\partial x_{2}}\right)\right. \\
& \left.+v \varepsilon_{22}\left(\frac{\partial \dot{z}_{1}}{\partial x_{1}}-\varepsilon_{11} \frac{\partial V_{1}}{\partial x_{1}}\right)\right] .
\end{aligned}
$$

\section{References}

ABAQUS (1999). User's Guide and Theoretical Manual, Version 5.8, Hibbitt, Karlsson and Sorenson, Inc., Pawtucket, RI.

Anderson, T.L. (1985). Fracture Mechanics: Fundamentals and Applications, Second Edition, CRC Press Inc., Boca Raton, Florida.

Barbero, E.J. and Reddy, J.N. (1990). The jacobian derivative method for three-dimensional fracture mechanics. Communications in Applied Numerical Methods 6, 507-518.

Besterfield, G.H., Lawrence, M.A. and Belytschko, T. (1990). Brittle fracture reliability by probabilistic finite elements. ASCE Journal of Engineering Mechanics, 116, 642-659.

Besterfield, G.H. , Liu, W.K., Lawrence, M.A. and Belytschko, T. (1991). Fatigue crack growth reliability by probabilistic finite elements, Computer Methods in Applied Mechanics and Engineering, 86, 297-320.

Choi, K.K. and Haug, E.J. (1983). Shape design sensitivity analysis of elastic structures. Journal of Structural Mechanics 11, 231-269.

deLorenzi, H.G. (1985). Energy release rate calculations by the finite element method. Engineering Fracture Mechanics 21, 129-143.

deLorenzi, H.G. (1982). On the energy release rate and the $J$-integral for 3-D crack configurations. International Journal of Fracture 19, 183-193. 
Feijóo, R.A., Padra, C., Saliba, R., Taroco, E. and Vénere, M.J. (2000). Shape sensitivity analysis for energy release rate evaluations and its application to the study of three-dimensional cracked bodies. Computational Methods in Applied Mechanics and Engineering 188, 649-664.

Grigoriu, M., Saif, M.T.A., El-Borgi, S. and Ingraffea, A. (1990). Mixed-mode fracture initiation and trajectory prediction under random stresses. International Journal of Fracture 45, 19-34.

Haber, R.B. and Koh, H.M. (1985). Explicit expressions for energy release rates using virtual crack extensions. International Journal of Numerical Methods in Engineering 21, 301-315.

Hasofer, A.M. and Lind, N.C. (1974). An exact and invariant first-order reliability format. Journal of Engineering Mechanics 100, 111-121.

Haug, E.J., Choi, K.K. and Komkov, V. (1986). Design Sensitivity Analysis of Structural Systems, Academic Press, New York.

Hwang, C.G., Wawrzynek, P.A., Tayebi, A.K. and Ingraffea, A.R. (1998). On the virtual crack extension method for calculation of the rates of energy release rate. Engineering Fracture Mechanics 59, 521-542.

Keum, D.J. and Kwak, B.M. (1992a). Calculation of stress intensity factors by sensitivity analysis with respect to change of boundary conditions. Computers and Structures 44, 63-69.

Keum, D.J. and Kwak, B.M. (1992b). Energy release rates of crack kinking by boundary sensitivity analysis. Engineering Fracture Mechanics 41, 833-841.

Li, F.Z., Shih, C.F. and Needleman A. (1985). A comparison of methods for calculating energy release rates. Engineering Fracture Mechanics 21, 405-421.

Lin, S.C. and Abel, J. (1988). Variational approach for a new direct-integration form of the virtual crack extension method. International Journal of Fracture 38, 217-235.

Liu, P.L. and Kiureghian, A.D. (1991). Optimization algorithms for structural reliability. Structural Safety 9, 161177.

Madsen, H.O., Krenk, S. and Lind, N.C. (1986). Methods of Structural Safety, Prentice-Hall, Inc., Englewood Cliffs, New Jersey.

Moran, B. and Shih, C.F. (1987). A general treatment of crack tip contour integrals. International Journal of Fracture 35, 295-310.

Provan, James, W. (1987). Probabilistic Fracture Mechanics and Reliability, Martinus Nijhoff Publ., Dordrecht, The Netherlands.

Rackwitz, R. and Fiessler, B. (1978). Structural reliability under combined random load sequence. Computers and Structures 9, 489-494.

Rahman, S. and Kim, J-S. (2001). Probabilistic fracture mechanics for nonlinear structures. International Journal of Pressure Vessels and Piping, 78, 261-269.

Rahman, S., Ghadiali, N., Paul, D. and Wilkowski, G. (1995). Probabilistic pipe fracture evaluations for leakrate-detection applications. NUREG/CR-6004, U.S. Nuclear Regulatory Commission, Washington, D.C.

Rahman, S. (1995). A stochastic model for elastic-plastic fracture analysis of circumferential through-wall-cracked pipes subject to bending. Engineering Fracture Mechanics, 52, 265-288.

Rahman, S. (2001). Probabilistic fracture mechanics by $J$-estimation and finite element methods. Engineering Fracture Mechanics, 68, 107-125.

Rice, J.R. (1968). A path independent integral and the approximate analysis of strain concentration by notches and cracks. Journal of Applied Mechanics 35, 379-386.

Shih, C.F., Moran, B. and Nakamura, T. (1986). Energy release rate along a three-dimensional crack front in a thermally stressed body. International Journal of Fracture 30, 79-102.

Taroco, E. (2000). Shape sensitivity analysis in linear elastic cracked structures. Computational Methods in Applied Mechanics and Engineering 188, 697-712. 